\title{
Global management of patients with knee osteoarthritis begins with quality of life assessment: a systematic review
}

\author{
Marianna Vitaloni ${ }^{1^{*}+} \mathbb{D}$, Angie Botto-van Bemden ${ }^{2^{*}+}$, Rosa Maya Sciortino Contreras ${ }^{3}$, Deborah Scotton ${ }^{2}$, \\ Marco Bibas ${ }^{1}$, Maritza Quintero ${ }^{3}$, Jordi Monfort ${ }^{4}$, Xavier Carné ${ }^{5}$, Francisco de Abajo ${ }^{6}$, Elizabeth Oswald ${ }^{1}$, \\ Maria R. Cabot ${ }^{7}$, Marco Matucci ${ }^{8}$, Patrick du Souich ${ }^{9}$, Ingrid Möller ${ }^{10}$, Guy Eakin ${ }^{2}$ and Josep Verges ${ }^{1}$
}

\begin{abstract}
Background: Knee osteoarthritis (KOA) is a prevalent form of chronic joint disease associated with functional restrictions and pain. Activity limitations negatively impact social connectedness and psychological well-being, reducing the quality of life $(\mathrm{QoL})$ of patients. The purpose of this review is to summarize the existing information on QoL in KOA patients and share the reported individual factors, which may influence it.

Methods: We conducted a systematic review examining the literature up to JAN/2017 available at MEDLINE, EMBASE, Cochrane, and PsycINFO using KOA and QOL related keywords. Inclusion criteria were QOL compared to at least one demographic factor (e.g., age, gender), lifestyle factor (e.g., functional independence), or comorbidity factor (e.g., diabetes, obesity) and a control group. Analytical methods were not considered as part of the original design.

Results: A total of 610 articles were reviewed, of which 62 met inclusion criteria. Instruments used to measure QoL included: SF-36, EQ-5D, KOOS, WHOQOL, HAS, AIMS, NHP and JKOM. All studies reported worse QoL in KOA patients when compared to a control group. When females were compared to males, females reported worse QOL. Obesity as well as lower level of physical activity were reported with lower QoL scores. Knee self-management programs delivered by healthcare professionals improved QoL in patients with KOA. Educational level and higher total mindfulness were reported to improve QoL whereas poverty, psychological distress, depression and lacking familial relationships reduce it. Surgical KOA interventions resulted in good to excellent outcomes generally; although, results varied by age, weight, and depression.

Conclusion: KOA has a substantial impact on QoL. In KOA patients, QoL is also influenced by specific individual factors including gender, body weight, physical activity, mental health, and education. Importantly, education and management programs designed to support KOA patients report improved QoL. QoL data is a valuable tool providing health care professionals with a better comprehension of KOA disease to aid implementation of the most effective management plan.
\end{abstract}

Keywords: Osteoarthritis, Knee, Quality of life, Individual factors, Social Determinants of Health, Psychosocial factors, Patient advocacy organizations, Patient Centred

\footnotetext{
* Correspondence: mvitaloni@oafifoundation.com; avanbemden@arthritis.org

${ }^{\dagger}$ Marianna Vitaloni and Angie Botto-van Bemden contributed equally to this

work.

${ }^{1}$ Osteoarthritis Foundation International OAFI, Barcelona, Spain

${ }^{2}$ Arthritis Foundation, Atlanta, USA

Full list of author information is available at the end of the article
}

(c) The Author(s). 2019 Open Access This article is distributed under the terms of the Creative Commons Attribution 4.0 International License (http://creativecommons.org/licenses/by/4.0/), which permits unrestricted use, distribution, and reproduction in any medium, provided you give appropriate credit to the original author(s) and the source, provide a link to the Creative Commons license, and indicate if changes were made. The Creative Commons Public Domain Dedication waiver (http://creativecommons.org/publicdomain/zero/1.0/) applies to the data made available in this article, unless otherwise stated. 


\section{Background}

Knee Osteoarthritis (KOA) is one of the primary causes of pain and disability worldwide. The pain and disability are associated with functional restrictions, morphological changes in the subchondral bone, articular cartilage degeneration and damage to the surrounding soft tissue [1-3]. In addition to the structural and functional limitations caused by KOA, pain and disability from KOA also affect social connectedness, relationships and emotional well-being; subsequently, reducing quality of life (QoL) [4]. The goal of treatment has traditionally focused on reducing pain and improving function, yet healthcare providers are increasingly realizing the importance of ensuring implementation of psychosocial support to improve the health and overall well-being of KOA patients. Assessing QoL is an imperative first step in evaluating well-being, disease progression and intervention efficacy [5-8].

Notably, measurement of QoL in KOA is increasing in research and clinical practice, but it still is not routine [9]. As far as we know, this is the first systematic review summarizing existing studies results reporting QoL in KOA patients combined with individual factors such as demographics (e.g., age, gender), lifestyle (e.g., functional independence), or comorbidities (e.g., diabetes, obesity).

The purpose of this review is to provide an international resource summarizing available studies, which have reported individual factors affecting QoL in KOA patients. Our results aim to prompt incorporation of psychosocial assessment in management strategies. Patient organization representatives designed and executed this summary to prompt routine evaluation of such.

\section{Methods}

\section{Search strategy}

We identified original articles using electronic searches of MEDLINE, EMBASE, Cochrane and PsycINFO databases. Literature review start date was unrestricted and end date was January 23, 2017. Searches were not limited by language. However, no eligible study was found in non-English languages. The keywords used were "knee osteoarthritis" AND "quality of life" OR "life quality" OR "wellbeing" OR "well-being" OR "short form 36" OR "knee injury and osteoarthritis outcome score" OR "koos" OR "koos-qol" OR "euroqol" OR "assessment of quality of life" OR "qualitymetric" OR "whoqol-100" OR "quality of wellbeing" OR "rosser" OR "osteoarthritis quality of life scale" OR "osteoarthritis knee and hip quality of life" OR "arthritis impact measurement scale" and all shorter forms and variations. The systematic review was conducted following Preferred Reporting Items for Systematic Reviews and Meta-Analyses guidelines [9].

\section{Inclusion and exclusion criteria}

An example of the tag flowchart for inclusion and exclusion can be found in online Additional file 1: Figure S1.
Only abstracts or articles reporting original data on QoL of KOA patients were included. Inclusion criteria were QoL associated to one or more demographic factors (e.g., age, gender), lifestyle factors (e.g., functional independence), or comorbidity factors (e.g., diabetes, obesity) and compared with a reference population, or control group. The control group was composed of individuals without KOA. There were no other restrictions on the comparison control group. There was no restriction on age, gender, language, or year of publication. Review articles, protocols for clinical trials, commentaries, editorials, proceedings summaries, or instrument development summaries were excluded from this review. Articles that described unspecified knee pain, pre-intervention anterior cruciate ligament repair, hip osteoarthritis (OA), spine OA or any study that combined KOA patients with other cohorts of patients and did not collect, analyse, or report KOA-specific data separately (e.g., a population defined as "hip or KOA" or "hip and/or KOA") were also excluded. Three reviewers independently assessed each reference against pre-specified inclusion and exclusion criteria using a two-stage process: first, titles and abstracts, and, second, full-text articles. Any queries were resolved during a consensus meeting.

\section{Data extraction}

A single reviewer, using a pre-piloted extraction form, obtained the data for each eligible article. Study characteristics including publication details (author and year), participant characteristics (age, sex, body mass index [BMI] and number of participants in each group), instruments, treatments applied in the intervention and control groups, and a summary of main findings were extracted from each included study for subsequent review amongst all three reviewers.

\section{Quality appraisal}

The quality assessment of each article was based on a modified version of the Cochrane quality appraisal tool [10]. The individual assessment criteria are presented in Fig. 1. Strength of consistency between raters was not scored yet individual and average quality assessment results are included in online Additional file 2. One point was allocated for each of the 13 quality appraisal criteria. The maximum score was 13 (indicating high quality), with the lowest possible score being zero. The methodological quality of each study was rated as low (0-4 points), moderate (5-9 points), or high (10-13 points).

\section{Results}

Literature search results

A total of 9143 articles were initially identified (Fig. 2); 4863 articles from EMBASE, 2792 from PubMed, 1279 from Cochrane and 209 from PsycINFO. A total of 610 


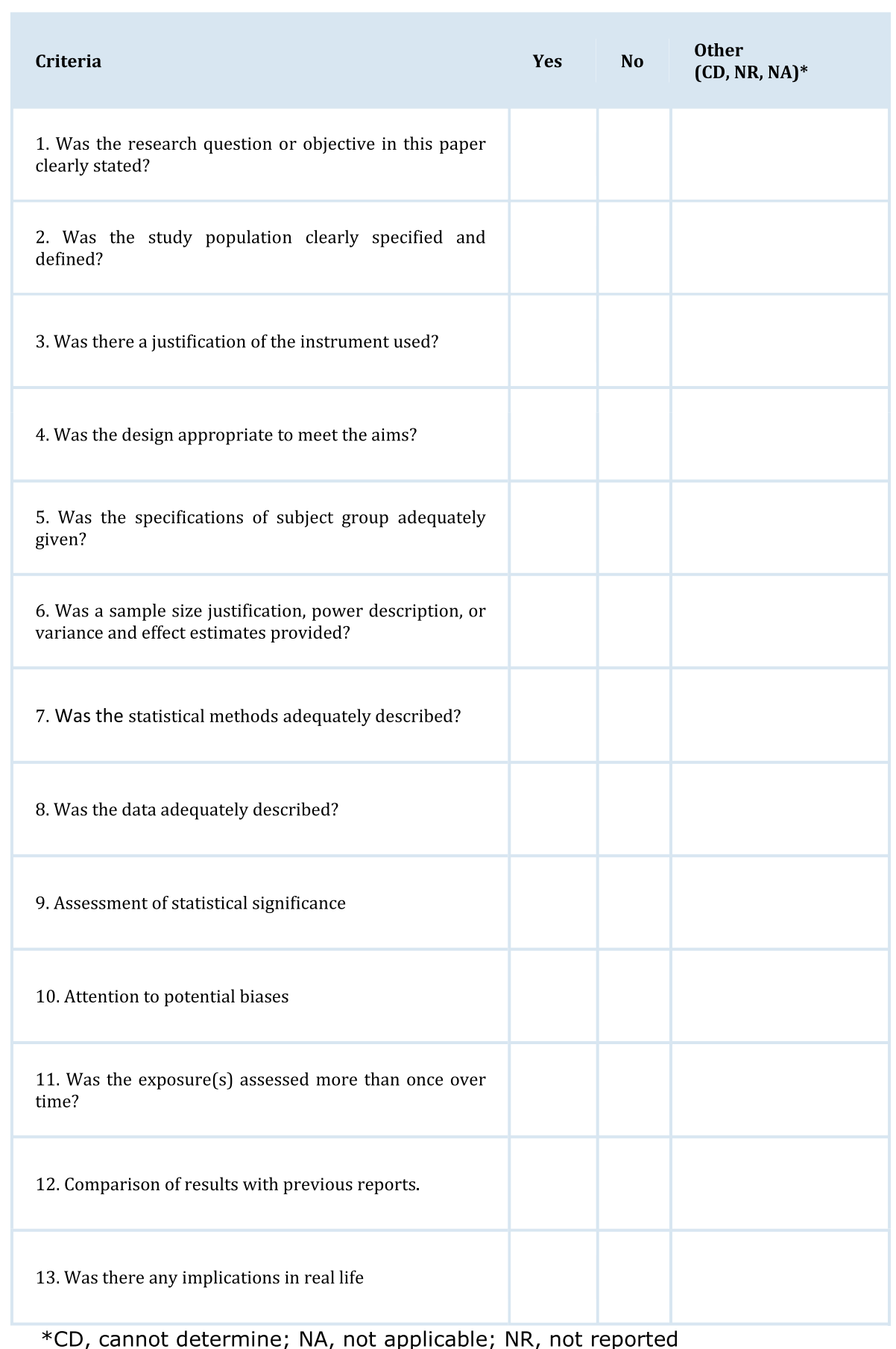

Fig. 1 Quality Appraisal tool. The quality appraisal tool is a modify version of the quality appraisal tool recommended by Cochrane. Three independent researchers scored the 13 items

articles were selected after initial title and abstract screening. After manual searches, full text review and removal of duplicates, 62 articles were included for final data extraction. Studies were labelled by first author and year of publication (Table 1). Year of publication ranged from 1995 to 2017.

\section{Characteristics of included studies}

Most of the studies were conducted in Europe $(n=20)$, followed by the North, Central and South America $(n=$ $16)$, Asia $(n=12)$ and other $(n=14)$. The $74 \%(n=46)$ of studies were cross-sectional in design, followed by other designs comprising $6 \%(n=4)$ prospective cohorts, $5 \%$ 


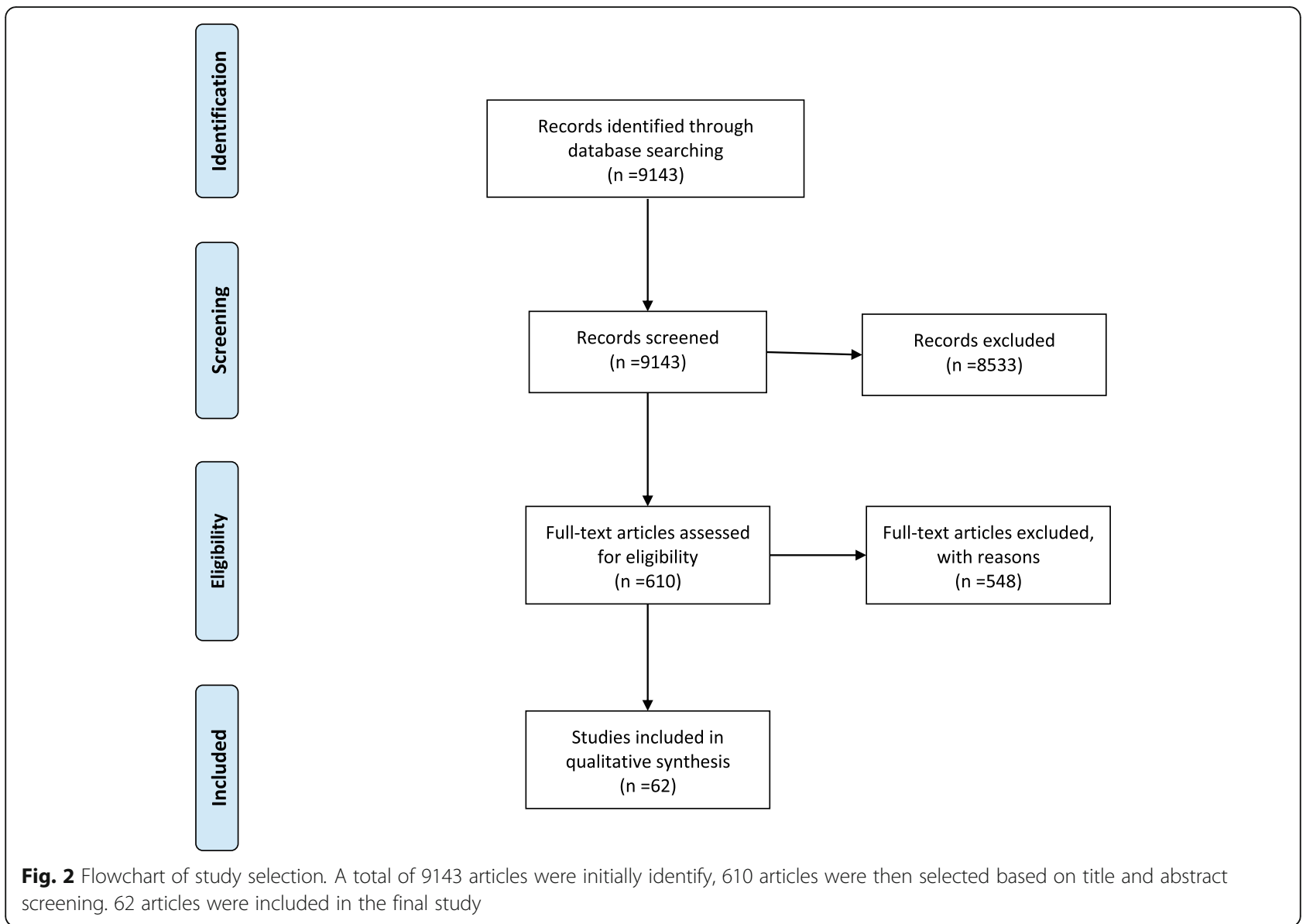

$(n=3)$ prospective, $3 \%(n=2)$ randomized controls trials, $(n=1)$ population based cohorts, $(n=1)$ retrospective, etc. The main instruments used to assess QoL were the SF-36 $(n=31)$ followed by EQ-5D $(n=6)$ (Table 1$)$. The results from the quality appraisal tool had all articles scoring moderate to high quality scores; no study scored lower than 8 points. Four $(6 \%)$ of the articles [25, 28, 54, 60] were classified as moderate quality, with the remaining 58 (94\%) articles classified as having high quality [11-24, 26, 27, 29-53, 55-59, 61-72].

\section{Characteristics of participants}

The total KOA population was 24,706 of which $93.4 \%$ $(n=23,079)$ were female [11-72]. 11 articles included only females $[19,26,28,37,41,43,53,55,56,60,63]$ and one article included only males [64]. When the male and female only articles were removed, the total KOA population still comprised $93.8 \%$ female. The mean number of KOA patients per study was 560 , with sample size varying between 12 [43] and 7977 [31] and the median number was 101. The mean age across all studies was 65 years (range 47 to 85 ) and 68.2 (range 56.9 to 71.1 ) years for those studies including only females $[19,26,28,37,41,43,53,55,56,60,63]$ and 50 (range 50 to 69) for the one study enrolling only males (Table 1) [64].

\section{QoL in patients with KOA versus reference populations}

Studies reported worse QoL for the KOA group, regardless of measurement used to assess QoL [12, 14, 22, 28, 31, 33, 38, 46, 53, 60, 61, 63, 65-67, 69]. Lower QoL scores were mostly reported with increasing age $[25,26$, $32,35,44,53]$ yet Jenkins reported higher QoL in older patients [57]. When QoL scores were compared based on gender, females with KOA reported worse QoL scores and psychosocial variables $[49,58,66]$. In online Additional file 3: Table S1 additional results are presented for studies comparing QoL of KOA patients with a reference population by instrument.

\section{QoL and healthy weight}

Weight was reported as effecting QoL in KOA patients [11, 20, 46, 67, 70]. Vulcano, Elbaz and Visser reported a higher BMI aggravated KOA patient symptoms [11, 46, 51]. Gomez-Neto found a negative impact on functional capacity in obese KOA patients; however, found no difference reported in QoL [20]. de Leeuw, reported that the median 
Table 1 Overview of studies reporting QoL in patients with KOA

\begin{tabular}{|c|c|c|c|c|c|c|c|c|}
\hline References & Country & Study design & $\begin{array}{l}\text { QoL } \\
\text { instrument }\end{array}$ & $\begin{array}{l}\text { Total } \\
\text { Sample } \\
\text { Size }\end{array}$ & $\begin{array}{l}\text { Control } \\
\text { Population }\end{array}$ & KOA Patients & $\begin{array}{l}\text { Mean } \\
\text { age }\end{array}$ & Gender Distribution \\
\hline \multirow{2}{*}{$\begin{array}{l}\text { Cuzdan, } 2017 \\
\text { [11] }\end{array}$} & \multirow[t]{2}{*}{ Turkey } & \multirow{2}{*}{$\begin{array}{l}\text { Cross- } \\
\text { Sectional }\end{array}$} & \multirow[t]{2}{*}{ SF-36 } & \multirow[t]{2}{*}{85} & \multirow[t]{2}{*}{25} & \multirow[t]{2}{*}{60} & \multirow[t]{2}{*}{65.79} & Knee OA: 57 female; 3 male \\
\hline & & & & & & & & Control: 13 female; 12 male \\
\hline \multirow{2}{*}{$\begin{array}{l}\text { Elbaz, } 2017 \\
{[12]}\end{array}$} & \multirow[t]{2}{*}{ Israel } & \multirow{2}{*}{$\begin{array}{l}\text { Prospective } \\
\text { observational }\end{array}$} & \multirow[t]{2}{*}{ SF-36 } & \multirow[t]{2}{*}{93} & \multirow[t]{2}{*}{30} & \multirow[t]{2}{*}{63} & \multirow[t]{2}{*}{64.2} & Knee OA: 41 female; 22 male \\
\hline & & & & & & & & Control: 9 female; 21 male \\
\hline \multirow[t]{2}{*}{ Lee, 2017 [13] } & \multirow[t]{2}{*}{ USA } & \multirow{2}{*}{$\begin{array}{l}\text { Cross- } \\
\text { Sectional }\end{array}$} & \multirow[t]{2}{*}{ SF-36 } & \multirow[t]{2}{*}{120} & \multirow[t]{2}{*}{40} & \multirow[t]{2}{*}{80} & \multirow[t]{2}{*}{60.3} & Knee OA: 61 female; 19 male \\
\hline & & & & & & & & Control: NA \\
\hline \multirow{2}{*}{$\begin{array}{l}\text { Rundell, } 2017 \\
\text { [14] }\end{array}$} & \multirow[t]{2}{*}{ USA } & \multirow[t]{2}{*}{ Prospective } & \multirow[t]{2}{*}{$E Q-5 D$} & \multirow[t]{2}{*}{5155} & \multirow[t]{2}{*}{4711} & 368 & 75.3 & Knee OA: 272 female; 96 male \\
\hline & & & & & & & & Control 3017 female; 1694 male \\
\hline $\begin{array}{l}\text { Wright, } 2017 \\
\text { [15] }\end{array}$ & Australia & $\begin{array}{l}\text { Cross- } \\
\text { Sectional }\end{array}$ & SF-36 & 120 & 40 & 80 & 64 & Knee OA: 44 female; 36 male \\
\hline $\begin{array}{l}\text { Araujo, } 2016 \\
{[16]}\end{array}$ & Brazil & $\begin{array}{l}\text { Cross- } \\
\text { Sectional }\end{array}$ & SF-12 & 93 & & 93 & 60 & $\begin{array}{l}\text { Knee OA: } 69 \text { female; } 24 \text { male } \\
\text { Control: } 24 \text { female; } 16 \text { male }\end{array}$ \\
\hline $\begin{array}{l}\text { Bokaeian, } \\
2016[17]\end{array}$ & Iran & $\begin{array}{l}\text { Randomized } \\
\text { clinical trial }\end{array}$ & WOMAC & 28 & & 28 & 52.9 & Knee OA: 25 female; 2 male \\
\hline Cho, 2016 [18] & $\begin{array}{l}\text { Republic of } \\
\text { Korea }\end{array}$ & $\begin{array}{l}\text { Prospective } \\
\text { cohort study }\end{array}$ & SF-36 & 681 & & & 71.9 & Knee OA: 383 female; 298 male \\
\hline $\begin{array}{l}\text { Kaban, } 2016 \\
{[19]}\end{array}$ & Turkey & $\begin{array}{l}\text { Cross- } \\
\text { Sectional }\end{array}$ & SF-36 & 63 & 21 & 42 & 56.86 & All female \\
\hline $\begin{array}{l}\text { Gomes-Neto, } \\
2016 \text { [20] }\end{array}$ & Brazil & $\begin{array}{l}\text { Cross- } \\
\text { Sectional }\end{array}$ & SF-36 & 35 & & 35 & 66.57 & Knee OA: 29 female; 6 male \\
\hline $\begin{array}{l}\text { Khatib, } 2016 \\
\text { [21] }\end{array}$ & Australia & $\begin{array}{l}\text { Cross- } \\
\text { Sectional }\end{array}$ & $\begin{array}{l}\text { Tot. EQ } \\
\text { (adjusted } \\
\text { from EQ-5D- } \\
5 \mathrm{~L} \text { ) }\end{array}$ & 2809 & & 2809 & 68 & Knee OA: 1740 female; 1069 male \\
\hline $\begin{array}{l}\text { Kiadaliri, } 2016 \\
{[22]}\end{array}$ & Sweden & $\begin{array}{l}\text { Population } \\
\text { based cohort } \\
\text { study }\end{array}$ & EQ-5D & 1501 & 744 & 402 & 71.5 & $\begin{array}{l}\text { Group } 1 \text { (reference group having } \\
\text { neither knee pain nor } \\
\text { radiographic or clinically-defined } \\
\text { knee OA) } 469 \text { female; } 275 \text { male }\end{array}$ \\
\hline & & & & & & & & $\begin{array}{l}\text { Group } 2 \text { (knee pain with-out OA) } \\
119 \text { female; } 50 \text { male }\end{array}$ \\
\hline & & & & & & & & $\begin{array}{l}\text { Group } 3 \text { (kne epain with OA) } 256 \\
\text { female; } 146 \text { male }\end{array}$ \\
\hline $\begin{array}{l}\text { Kiadaliri, } 2016 \\
{[23]^{\mathrm{a}}}\end{array}$ & Sweden & Retrospective & EQ-5D & $\begin{array}{l}\text { First stage } \\
7402 ;\end{array}$ & & $\begin{array}{l}\text { The number of } \\
\text { people diagnosis }\end{array}$ & 69.4 & $\begin{array}{l}\text { First stage } 4604 \text { female; } 2798 \\
\text { male }\end{array}$ \\
\hline & & & & $\begin{array}{l}\text { second } \\
\text { stage } \\
1527\end{array}$ & & not specified & & $\begin{array}{l}\text { Second stage } 977 \text { female; } 550 \\
\text { male }\end{array}$ \\
\hline Oishi, 2016 & Japan & Cross- & KOOS & 963 & & 397 & 54.33 & Total: 595 female; 368 male \\
\hline & & & & & & & & Knee OA: 299 female; 98 male \\
\hline $\begin{array}{l}\text { Sarumathy, } \\
2016[25]\end{array}$ & India & $\begin{array}{l}\text { Prospective } \\
\text { study }\end{array}$ & SF-36 & 74 & & 74 & 51.7 & Knee OA: 55 female; 19 male \\
\hline $\begin{array}{l}\text { Cavalcante, } \\
2015[26]\end{array}$ & Brazil & $\begin{array}{l}\text { Cross- } \\
\text { Sectional }\end{array}$ & WHOQOL & 90 & 40 & 50 & 67 & All female \\
\hline Fang, 2015 & Taiwan & Population & SF-12 & 901 & 441 & 460 & 74.04 & Total: 492 female; 409 male \\
\hline & & $\begin{array}{l}\text { Dased } \\
\text { study }\end{array}$ & & & & & & Knee OA: 232 female; 209 male \\
\hline $\begin{array}{l}\text { Ferreira, } 2015 \\
\text { [28] }\end{array}$ & Brazil & $\begin{array}{l}\text { Cross- } \\
\text { Sectional }\end{array}$ & SF-36 & 75 & 35 & 40 & 68.36 & All female \\
\hline $\begin{array}{l}\text { Kawano, } 2015 \\
{[29]}\end{array}$ & Brazil & $\begin{array}{l}\text { Cross- } \\
\text { Sectional }\end{array}$ & SF-36 & 93 & & 93 & 61.2 & Knee OA: 69 females; 24 male \\
\hline Kim, 2015 [30] & Korea & $\begin{array}{l}\text { Cross- } \\
\text { Sectional }\end{array}$ & EQ-5D & 2165 & & 2165 & 67.73 & Knee OA: 1458 female; 707 male \\
\hline
\end{tabular}


Table 1 Overview of studies reporting QoL in patients with KOA (Continued)

\begin{tabular}{|c|c|c|c|c|c|c|c|c|}
\hline References & Country & Study design & $\begin{array}{l}\text { QoL } \\
\text { instrument }\end{array}$ & $\begin{array}{l}\text { Total } \\
\text { Sample } \\
\text { Size }\end{array}$ & $\begin{array}{l}\text { Control } \\
\text { Population }\end{array}$ & KOA Patients & $\begin{array}{l}\text { Mean } \\
\text { age }\end{array}$ & Gender Distribution \\
\hline Lee, 2015 [31] & $\begin{array}{l}\text { South } \\
\text { Korea }\end{array}$ & $\begin{array}{l}\text { Cross- } \\
\text { Sectional }\end{array}$ & EQ-5D & 7977 & & 7977 & 61.5 & Knee OA: 5448 female; 4064 male \\
\hline $\begin{array}{l}\text { Pang, } 2015 \\
\text { [32] }\end{array}$ & China & $\begin{array}{l}\text { Cross- } \\
\text { Sectional }\end{array}$ & SF-36 & 466 & & 466 & 56.56 & Knee OA: 382 female; 84 male \\
\hline \multirow{2}{*}{$\begin{array}{l}\text { Rakel, } 2015 \\
\text { [33] }\end{array}$} & \multirow[t]{2}{*}{ USA } & \multirow{2}{*}{$\begin{array}{l}\text { Cross- } \\
\text { Sectional }\end{array}$} & \multirow[t]{2}{*}{ SF-36 } & \multirow[t]{2}{*}{100} & \multirow[t]{2}{*}{25} & \multirow[t]{2}{*}{75} & \multirow[t]{2}{*}{56} & Knee OA: 46 female; 29 male \\
\hline & & & & & & & & Control: 15 female; 10 male \\
\hline $\begin{array}{l}\text { Reid, } 2015 \\
\text { [34] }\end{array}$ & USA & $\begin{array}{l}\text { Randomized } \\
\text { controlled } \\
\text { trial }\end{array}$ & SF-36 & 190 & & 190 & 60.2 & Knee OA: 132 female; 58 male \\
\hline $\begin{array}{l}\text { Tsonga, } 2015 \\
\text { [35] }\end{array}$ & Greece & Longitudinal & SF-36 & 68 & & 68 & 73 & Knee OA: 57 female; 11 male \\
\hline \multirow{3}{*}{$\begin{array}{l}\text { Visser, } 2015 \\
{[36]}\end{array}$} & \multirow[t]{3}{*}{ Netherlands } & \multirow{3}{*}{$\begin{array}{l}\text { Cross- } \\
\text { Sectional }\end{array}$} & \multirow[t]{3}{*}{ SF-36 } & \multirow[t]{3}{*}{1262} & \multirow[t]{3}{*}{1060} & \multirow[t]{3}{*}{205} & \multirow[t]{3}{*}{56} & Total: 707 female; 578 male \\
\hline & & & & & & & & Knee OA: 125 female; 80 male \\
\hline & & & & & & & & Control: 583 female; 477 male \\
\hline $\begin{array}{l}\text { Alburquerque- } \\
\text { garcía, } 2015 \\
\text { [37] }\end{array}$ & Spain & $\begin{array}{l}\text { Cross- } \\
\text { Sectional }\end{array}$ & SF-36 & 36 & 18 & 18 & 85 & All female \\
\hline \multirow{2}{*}{$\begin{array}{l}\text { Alkan, } 2014 \\
\text { [38] }\end{array}$} & \multirow[t]{2}{*}{ Turkey } & \multirow{2}{*}{$\begin{array}{l}\text { Cross- } \\
\text { Sectional }\end{array}$} & \multirow[t]{2}{*}{ SF-36 } & \multirow[t]{2}{*}{152} & \multirow[t]{2}{*}{40} & \multirow[t]{2}{*}{112} & \multirow[t]{2}{*}{60} & Knee OA: 85 female; 27 male \\
\hline & & & & & & & & Control: 30 female 10 male \\
\hline $\begin{array}{l}\text { Forkel, } 2014 \\
\text { [39] }\end{array}$ & Germany & $\begin{array}{l}\text { Cross- } \\
\text { Sectional }\end{array}$ & KOOS & 22 & & 22 & 47 & Knee OA: 17 female; 6 male \\
\hline $\begin{array}{l}\text { Jahnke, } 2014 \\
\text { [40] }\end{array}$ & Germany & $\begin{array}{l}\text { Cross- } \\
\text { Sectional }\end{array}$ & HAS & 159 & & 159 & 63.5 & Knee OA: 75 female; 84 male \\
\hline $\begin{array}{l}\text { Marks, } 2014 \\
\text { [41] }\end{array}$ & USA & $\begin{array}{l}\text { Cross- } \\
\text { Sectional }\end{array}$ & AIMS & 21 & & 21 & 70.8 & All female \\
\hline $\begin{array}{l}\text { Pérez-Prieto, } \\
2014 \text { [42] }\end{array}$ & Spain & $\begin{array}{l}\text { Prospective } \\
\text { cohort study }\end{array}$ & SF-36 & 716 & & 716 & 72 & Knee OA: 421 female; 295 male \\
\hline Reis, 2014 [43] & Brazil & $\begin{array}{l}\text { Cross- } \\
\text { Sectional }\end{array}$ & WHOQOL & 12 & & 12 & 67.25 & All female \\
\hline $\begin{array}{l}\text { Alentorn, } \\
2013 \text { [44] }\end{array}$ & Spain & $\begin{array}{l}\text { Cross- } \\
\text { Sectional }\end{array}$ & SF-36 & 391 & & 391 & 70.7 & Knee OA: 303 female; 89 male \\
\hline $\begin{array}{l}\text { Clement, } 2013 \\
\text { [45] }\end{array}$ & UK & $\begin{array}{l}\text { Cross- } \\
\text { Sectional }\end{array}$ & SF-12 & 996 & & 996 & 70.32 & Knee OA: 545 female; 421 male \\
\hline $\begin{array}{l}\text { Vulcano, } 2013 \\
\text { [46] }\end{array}$ & USA & $\begin{array}{l}\text { Prospective } \\
\text { cohort study }\end{array}$ & SF-36 & 4732 & & 4732 & 66.88 & Knee OA: 2881 female; 1851 male \\
\hline $\begin{array}{l}\text { Williams, } 2013 \\
\text { [47] }\end{array}$ & UK & $\begin{array}{l}\text { Cross- } \\
\text { Sectional }\end{array}$ & EQ-5D & 2456 & & 2456 & 71.4 & Knee OA: 1494 female; 962 male \\
\hline $\begin{array}{l}\text { Coleman, } \\
2012 \text { [48] }\end{array}$ & Australia & $\begin{array}{l}\text { Cross- } \\
\text { Sectional }\end{array}$ & SF-36 & 146 & & 146 & 65 & Knee OA: 109 female; 37 male \\
\hline $\begin{array}{l}\text { Gonçalves, } \\
2012 \text { [49] }\end{array}$ & Portugal & $\begin{array}{l}\text { Cross- } \\
\text { Sectional }\end{array}$ & SF-36 & 136 & & 136 & 67.2 & Knee OA: 94 female; 42 male \\
\hline Lim, 2012 [50] & Philippine & $\begin{array}{l}\text { Cross- } \\
\text { Sectional }\end{array}$ & WOMAC & 90 & & & 70.14 & Knee OA: 68 female; 22 male \\
\hline $\begin{array}{l}\text { Elbaz, } 2011 \\
\text { [51] }\end{array}$ & Israel & $\begin{array}{l}\text { Cross- } \\
\text { Sectional }\end{array}$ & SF-36 & 1487 & & 1487 & 61.9 & Knee OA: 950 female; 537 male \\
\hline $\begin{array}{l}\text { Gonçalves, } \\
2011 \text { [52] }\end{array}$ & Portugal & $\begin{array}{l}\text { Cross- } \\
\text { Sectional }\end{array}$ & KOOS & 377 & & 377 & 67.8 & Knee OA: 282 females; 95 males \\
\hline $\begin{array}{l}\text { Norimatsu, } \\
2011 \text { [53] }\end{array}$ & Japan & $\begin{array}{l}\text { Prospective } \\
\text { population- } \\
\text { based cohort } \\
\text { study }\end{array}$ & $\begin{array}{l}\text { Japanese } \\
\text { Knee } \\
\text { Osteoarthritis } \\
\text { Measure } \\
\text { (JKOM) }\end{array}$ & 333 & & 333 & 64.2 & All female \\
\hline
\end{tabular}


Table 1 Overview of studies reporting QoL in patients with KOA (Continued)

\begin{tabular}{|c|c|c|c|c|c|c|c|c|}
\hline References & Country & Study design & $\begin{array}{l}\text { QoL } \\
\text { instrument }\end{array}$ & $\begin{array}{l}\text { Total } \\
\text { Sample } \\
\text { Size }\end{array}$ & $\begin{array}{l}\text { Control } \\
\text { Population }\end{array}$ & KOA Patients & $\begin{array}{l}\text { Mean } \\
\text { age }\end{array}$ & Gender Distribution \\
\hline $\begin{array}{l}\text { Ozcakir, } 2011 \\
{[54]}\end{array}$ & Turkey & $\begin{array}{l}\text { Cross- } \\
\text { Sectional }\end{array}$ & $\mathrm{NHP}$ & 100 & & 100 & 59.5 & Knee OA: 83 female; 17 male \\
\hline $\begin{array}{l}\text { Paker, } 2011 \\
{[55]}\end{array}$ & Turkey & $\begin{array}{l}\text { Cross- } \\
\text { Sectional }\end{array}$ & SF-36 & 75 & & 75 & 66.1 & All female \\
\hline $\begin{array}{l}\text { Foroughi, } \\
2010 \text { [56] }\end{array}$ & Australia & $\begin{array}{l}\text { Cross- } \\
\text { Sectional }\end{array}$ & SF-36 & 17 & & 17 & 66 & All female \\
\hline $\begin{array}{l}\text { Jenkins, } 2010 \\
\text { [57] }\end{array}$ & USA & $\begin{array}{l}\text { Cross- } \\
\text { Sectional }\end{array}$ & QLI-A & 75 & & 75 & 69 & Knee OA: 57 female; 18 male \\
\hline Kim, 2010 [58] & Korea & $\begin{array}{l}\text { Prospective } \\
\text { cohort study }\end{array}$ & WOMAC & 504 & & 504 & 70.2 & Knee OA: 274 female; 230 male \\
\hline $\begin{array}{l}\text { Muraki, } 2010 \\
\text { [59] }\end{array}$ & Japan & $\begin{array}{l}\text { Cross- } \\
\text { Sectional }\end{array}$ & SF-8 & 2126 & & 2126 & 68.9 & Knee OA: 1359 female; 767 male \\
\hline $\begin{array}{l}\text { Watanabe, } \\
2010[60]\end{array}$ & Japan & $\begin{array}{l}\text { Cross- } \\
\text { Sectional }\end{array}$ & $\begin{array}{l}\text { Japanese } \\
\text { Knee } \\
\text { Osteoarthritis } \\
\text { Measure } \\
\text { (JKOM) }\end{array}$ & 18 & & 18 & 67 & All female \\
\hline $\begin{array}{l}\text { Yildiz, } 2010 \\
{[61]}\end{array}$ & Turkey & $\begin{array}{l}\text { Cross- } \\
\text { Sectional }\end{array}$ & $\mathrm{NHP}$ & 140 & & 140 & 59.39 & Knee OA: 104 females; 36 males \\
\hline $\begin{array}{l}\text { Debi, } 2009 \\
\text { [62] }\end{array}$ & Israel & $\begin{array}{l}\text { Cross- } \\
\text { Sectional }\end{array}$ & SF-36 & 134 & & & 66.95 & Knee OA: 85 females; 49 males \\
\hline \multirow{2}{*}{$\begin{array}{l}\text { Imamura, } \\
2008 \text { [63] }\end{array}$} & \multirow[t]{2}{*}{ Brazil } & \multirow{2}{*}{$\begin{array}{l}\text { Cross- } \\
\text { Sectional }\end{array}$} & \multirow[t]{2}{*}{ SF-36 } & \multirow[t]{2}{*}{84} & \multirow[t]{2}{*}{22} & \multirow[t]{2}{*}{62} & 71.1 & \multirow[t]{2}{*}{ All female } \\
\hline & & & & & & & $\begin{array}{l}\text { Control } \\
68.95\end{array}$ & \\
\hline \multirow{2}{*}{$\begin{array}{l}\text { Liikavainio, } \\
2008 \text { [64] }\end{array}$} & \multirow[t]{2}{*}{ Finland } & \multirow{2}{*}{$\begin{array}{l}\text { Cross- } \\
\text { Sectional }\end{array}$} & \multirow[t]{2}{*}{ RAND-36 } & \multirow[t]{2}{*}{107} & \multirow[t]{2}{*}{53} & \multirow[t]{2}{*}{54} & 59 & \multirow[t]{2}{*}{ All male } \\
\hline & & & & & & & $\begin{array}{l}\text { Control } \\
59.24\end{array}$ & \\
\hline $\begin{array}{l}\text { Wang, } 2008 \\
{[65]}\end{array}$ & Germany & $\begin{array}{l}\text { Cross- } \\
\text { Sectional }\end{array}$ & SF-36 & 1009 & & 1009 & 48.5 & Knee OA: 620 female; 389 male \\
\hline $\begin{array}{l}\text { Nunez, } 2007 \\
{[66]}\end{array}$ & Spain & $\begin{array}{l}\text { Cross- } \\
\text { Sectional }\end{array}$ & SF-36 & 100 & & 100 & 71.2 & Knee OA: 71 female; 29 male \\
\hline \multirow{2}{*}{$\begin{array}{l}\text { Salaffi, } 2005 \\
\text { [67] }\end{array}$} & \multirow[t]{2}{*}{ Italy } & \multirow{2}{*}{$\begin{array}{l}\text { Cross- } \\
\text { Sectional }\end{array}$} & \multirow[t]{2}{*}{ SF-36 } & \multirow[t]{2}{*}{264} & & KneeOA 108 & \multirow[t]{2}{*}{65.7} & Knee OA: 64 female; 44 male \\
\hline & & & & & & $\begin{array}{l}\text { Knee OA + Hip } \\
\text { OA } 51\end{array}$ & & $\begin{array}{l}\text { Knee OA + Hip OA: } 32 \text { female; } 19 \\
\text { male }\end{array}$ \\
\hline $\begin{array}{l}\text { Chacón, } 2004 \\
\text { [68] }\end{array}$ & Venezuela & $\begin{array}{l}\text { Cross- } \\
\text { Sectional }\end{array}$ & AIMS & 126 & & 126 & 64 & Knee OA: 106 female; 20 male \\
\hline $\begin{array}{l}\text { Lam, } 2000 \\
{[69]}\end{array}$ & China & $\begin{array}{l}\text { Cross- } \\
\text { Sectional } \\
\text { case-control } \\
\text { study }\end{array}$ & $\begin{array}{l}\text { COOP/ } \\
\text { WONCA }\end{array}$ & 760 & & 760 & 57.6 & Knee OA: 538 female; 222 male \\
\hline $\begin{array}{l}\text { de Leeuw, } \\
1998[70]\end{array}$ & UK & $\begin{array}{l}\text { Prospective } \\
\text { trial }\end{array}$ & $\begin{array}{l}\text { Rosser Index } \\
\text { Matrix }\end{array}$ & 101 & & 101 & 71.5 & Knee OA: 62 female; 39 male \\
\hline $\begin{array}{l}\text { Donnell, } 1998 \\
\text { [71] }\end{array}$ & France & $\begin{array}{l}\text { Cross- }^{-} \\
\text {Sectional }\end{array}$ & $\begin{array}{l}\text { Rosser Index } \\
\text { Matrix }\end{array}$ & 221 & & 221 & $\begin{array}{l}\text { No } \\
\text { specified }\end{array}$ & Knee OA: 174 female; 47 male \\
\hline Ries, 1995 [72] & USA & $\begin{array}{l}\text { Cross- } \\
\text { Sectional }\end{array}$ & AIMS & 47 & & 47 & 69.2 & Knee OA: 44 female; 3 male \\
\hline
\end{tabular}


pre-operative QoL scores for obese patients were significantly lower than for non-obese [70].

\section{QoL and Physical Activity}

Calvacante et al. found that KOA in older women can promote a decline in time spent performing physical activity and functional fitness with decline in QoL and an increase in sitting time [26]. Wantanabe, reported reduced physical activity resulted in worse QoL and also reported too much physical activity may exacerbate the development of KOA [60]. Strength training (ST) had a positive effect in KOA patients [17]. Reid found that muscle power is an independent determinant of QoL in KOA [34].

\section{QoL and educational programs}

Coleman evaluated a self-management educational program delivered by health care professionals, reporting an improved QoL in KOA patients assessed at 8 weeks and 6 months based on WOMAC and SF-36 measures [48].

\section{QoL and psychosocial factors}

Higher educational level and higher total mindfulness scores were reported to improve patients' perception of QoL [13, 23, 28]. Alkan et al. reported that approximately $70 \%$ of the study participants had low-middle education, resulting in poor QoL in this group [38]. Another study reported lower Health-Related Quality of Life (HRQL) scores for KOA females with chronic physical or mental health conditions [62]. Poverty also worsened QoL in KOA patients [45]. Psychological distress and depression worsened QoL [23, 28, 42, 54]. Patients lacking familial relationships reported worse QoL [50].

\section{QoL and surgical interventions}

The majority of QoL findings with surgical intervention were reported as dependents of demographic variables, which showed an effect on QoL after surgical outcomes. Williams et al. [47], for example, reported that patient satisfaction was lower among patients younger than 55 years of age compared to older patients. Vulcano, reported a higher BMI was associated with worse outcomes [46]. Perez-Prieto, reported patients with depression had less improvement that non-depressed patients after surgical intervention, reporting lower QoL scores [42]. Lower socioeconomic groups undergoing surgical intervention also reported worse QoL [45]. Patients participating in sports pre- and post- surgical intervention reported higher QoL scores [40].

\section{Discussion}

This systematic review aimed to broaden the amount of QoL data available for summarizing, using less stringent search criteria; for example, inclusion of articles irrespective of QoL being the primary endpoint. This review included surgical and non-surgical data including QoL measurement.

The broad search strategy identified 62 articles as reporting information on QoL associated to one other factor (demographic, lifestyle, or comorbidity) between KOA patients and control patients. Article details are presented in Table 1. The quality appraisal tool revealed all studies as moderate to high quality yet caution should be taken in the interpretation of findings, as this tool may not discern scientific \& analytic rigour assessing QoL but scores prioritizing criteria description. For example, describing sample size, potential biases, etc. would be sufficient to permit a high score even with low analytic rigour.

It is well known that patient characteristics influence QoL. In this summary, increasing age showed worse QoL in most studies; however, in an older age group of KOA patients awaiting total knee replacement better QoL scores were reported [57]. This was a convenience sample with predominantly married, white females in a higher socioeconomic class which may have biased the results for higher QoL scores. Notably, prior reports have demonstrated that younger populations have higher expectations in terms of QoL as they expect to perform better on many activities of daily living, work and recreation [73]. It is also known that gender effects QoL in KOA patients, as reported here in a prospective aging cohort study by Kim et al. [58] the percentage of males and females were similar. Females reported worse QoL than males with KOA and females had significantly higher risk for belonging to the worst quartile for all WOMAC subscales compared to males regardless of KOA presence after adjustment of age, BMI and OA severity. Goncalves reported similar results in most SF-36 subscales [49]. Most studies in this summary comprised an above average percentage of females beyond KOA population norms which may have revealed bias toward lower QoL scores if a risk assessment for bias had been performed.

Similar to recent reports, lifestyle factors and common social determinants of health, such as unhealthy weight, low physical activity, low socioeconomic and education levels were found to have a negative impact on QoL in KOA patients $[20,38,45,60]$. Understandably quantitative data is lacking in this summary, yet qualitative summaries and recent quantitative reports emphasize the importance of assessing these factors and implementing a whole person approach to healthcare. Health promotion and self-management strategies addressing unhealthy weight and low levels of physical activity may improve QoL. Obesity data highlights a gap and opportunity to improve KOA QoL scores by incorporating dietary guidelines and nutritional education [20, 74]. 
Programs where patients participate in education and supervised exercise delivered by trained physiotherapists can improve physical activity and QoL. Moreover, exercise therapy may postpone total joint replacement in patients with OA [75]. Low educational level increases the chance of having $\mathrm{OA}$, and results in decreases in patients' self-perception of QoL [76]. This relationship between education and the socioeconomic level is well recognized as individuals with lower education levels are generally relegated to manual or repetitive occupational activities increasing their risk of OA.

QoL is a powerful indicator to consider when implementing and evaluating OA management programs. QoL is best monitored and reassessed in the short and long term to ensure effectiveness of interventions. Available data on QoL interventions can be customized considering individual characteristics to improve the factors open to modulation such as weight, physical activity, emotional health and social connectedness. Management strategies may be optimized by adapting to patientspecific needs with a multimodal personalized OA management plan grounded on evidence-based therapies for whole person care.

\section{Limitations}

There are limitations to acknowledge and use as opportunities to improve quality of future OA research and reporting to have a more meaningful impact for OA patients. The database search did not include SPORTDiscus or Cumulative Index to Nursing and Allied Health Literature (CINAHL) which may have provided additional QoL study data, particularly assessing QoL related to healthy weight, physical activity, educational and psychosocial factors. The search was limited to 2017 and additional studies may have been published thereafter reporting on QoL in KOA patients. Caution should be used when interpreting these findings, as studies were included which may not have been scientifically rigorous upon review, yet methodological reporting sufficed to meet the data extraction criteria. For example, a moderate to high score could be obtained for providing an adequate description of most criteria even if the sample size and statistical methods weren't methodologically and analytically rigorous. Articles were included whether power analysis was performed on QoL as the primary outcome or not; thus, sample size may not have been sufficient to find differences when not powered for QoL as the primary outcome. Risk of bias was not calculated. The methodological heterogeneity of the studies limited unbiased comparisons and quantitative syntheses was not permissible.

\section{Conclusion}

KOA studies routinely include pain and function scores yet haven't routinely included psychosocial variables assessing QoL, which also influences how patients feel, function, and survive [77]. Unfortunately, there is no consensus on the core domains of QoL. Ensuring a standard QoL assessment is implemented, as routine care globally is imperative for healthcare professionals to gain a better understanding of OA disease whilst ensuring most optimal management.

This study was coordinated by patient organizations and previously promoted at the 2019 OARSI Annual Meeting advocating for routine assessment and on-going evaluation of QoL with implementation of a single agreed upon QoL measure [78]. Future KOA QoL research should combine efforts globally and focus on consistent quantitative and qualitative measures for more meaningful impact and interpretation.

\section{Supplementary information}

Supplementary information accompanies this paper at https://doi.org/10. 1186/s12891-019-2895-3.

Additional file 1: Figure S1. Flowchart used in the selection of the articles included in the study. The flowchart shows the sequence of criteria followed for the selection of the articles included in the study.

Additional file 2. Quality appraisal report results obtained after the evaluation of articles quality based on the Cochrane modified instrument rated by three independent reviewers and the mean value of the three scores.

Additional file 3: Table S1. Quality of life of KOA patients vs. reference population by instrument used. Table comparing the variable values in KOA patient's population with reference populations and statistical significance analysis. The data are organized

by article.

\section{Abbreviations}

CINAHL: Cumulative Index to Nursing and Allied Health Literature; BMI: Body mass index; HAS: Heidelberg Sports Activity Score; HRQL: Health-Related Quality of Life; KOA: Knee Osteoarthritis; OA: Osteoarthritis; QoL: Quality of life; ST: Strengthening training; TKR: Total knee replacement;

UKA: Uncompartmentalized knee arthroplasty

\section{Acknowledgments}

We would like to thank all the patients and volunteers who collaborate and continually support the activity and mission of our organizations.

\section{Authors' contributions}

$\mathrm{JV}$ conceived and designed the study and was in charge of overall direction and planning; GE, MB and EO helped supervise the project; MV and ABvB executed and lead completion of the project; RMSC wrote the manuscript with support and revisions from MV, ABvB and JV; EO, MB, GE, FdA, MQ, JM, $I M, X C, P d S$ and MM supported the development of the protocol of study; MRC, MV and RMSC performed the quality appraisal; DS, MV, ABVB, RMSC revised the manuscript. DS analysed the gender and age-related results; $A B \vee B, M V$, JV supervised the findings of this work. All authors discussed the results and have read and approved the final manuscript.

\section{Funding}

The authors did not receive any funding for the volunteer research work which produced the submitted manuscript.

Availability of data and materials

The source of data has been included in the manuscript.

Ethics approval and consent to participate

Not applicable. 


\section{Consent for publication}

Not applicable.

\section{Competing interests}

Dr. Möller declares to be a member of the editorial board of this journal.

\section{Author details}

'Osteoarthritis Foundation International OAFI, Barcelona, Spain. ${ }^{2}$ Arthritis Foundation, Atlanta, USA. ${ }^{3}$ De los Andes University, Merida, Venezuela. ${ }^{4}$ Rheumatology Service, Del Mar Hospital, Barcelona, Spain. ${ }^{5}$ Clinical Pharmacology Department, Clinic Hospital, Barcelona, Spain. ${ }^{6}$ Clinical Pharmacology Department, Alcalá University, Madrid, Spain. 'Faculty of Nursing, Clinic Hospital, Barcelona, Spain. ${ }^{8}$ Rheumatology Service, University of Florence, Florence, Italy. ${ }^{9}$ University of Montreal, Montreal, Canada. ${ }^{10} \mathrm{Poal}$ Institute, University of Barcelona, Barcelona, Spain.

Received: 31 May 2019 Accepted: 14 October 2019

Published online: 27 October 2019

\section{References}

1. Heiden TL, Lloyd DG, Ackland TR. Knee extension and flexion weakness in people with knee osteoarthritis: is antagonist cocontraction a factor? Orthop Sports Phys Ther. 2009;39(11):807-15

2. Di Cesare PE, Abramson SB, Samuels J. Pathogenesis of osteoarthritis. In: Firestein GS, Budd RC, Haris ED, Mclnnes B, Ruddy S, Sergent JS, editors. Kelley's textbook of rheumatology (vol II). Philadelphia: WB Saunders; 2009 p. $1525-6$.

3. Fernandes L, Hagen KB, Bijlsma JW, Andreassen O, Christensen P, Conaghan PG, et al. EULAR recommendations for the nonpharmacological core management of hip and knee osteoarthritis. Ann Rheum Dis. 2013;72:1125-35.

4. Corti MC, Rigon C. Epidemiology of osteoarthritis: prevalence, risk factors and functional impact. Aging Clin Exp Res. 2003;15:359-63.

5. Dillon CF, Rasch EK, Gu Q, Hirsch R. Prevalence of knee osteoarthritis in the United States: arthritis data from the third National Health and nutrition examination survey 1991-94. J Rheumatol. 2006;33(11):2271-9 Epub 2006 Oct 1

6. Cecchi F, Mannoni A, Molino-Lova R, Ceppatelli S, Benvenuti E, Bandinelli S, Lauretani F, Macchi C, Ferrucci L. Epidemiology of hip and knee pain in a community based sample of Italian persons aged 65 and older. Osteoarthr Cartil. 2008;16:1039-46

7. Lawrence RC, Felson DT, Helmick CG, Arnold LM, Choi H, Deyo RA, Gabriel S, Hirsch R, Hochberg MC, Hunder GG, Jordan JM, Katz JN, Kremers HM, Wolfe F, National Arthritis Data Workgroup. Estimates of the prevalence of arthritis and other rheumatic conditions in the United States: part II. Arthritis Rheum. 2008;58:26-35.

8. de Rezende MU, de Campos GC, Pailo AF, Current concepts in osteoarthritis. Acta Ortopedica Brasileira. Acta Ortop Bras. 2013;21(2):120-2. https://doi.org/ 10.1590/S1413-78522013000200010

9. Liberati A, Altman DG, Tetzlaff J, Mulrow C, Gøtzsche PC, loannidis JP. The PRISMA statement for reporting systematic reviews and meta-analyses of studies that evaluate healthcare interventions: explanation and elaboration. BMJ. 2009:339:b2700

10. National Institutes of Health. Study Quality Assessment Tools. Quality assessment tool for observational cohort and cross-sectional studies. 2014 Available from: https://www.nhlbi.nih.gov/health-topics/study-qualityassessment-tools.

11. Cuzdan Coskun N, Ay S, Evcik FD, Oztuna D. Adiponectin: is it a biomarker for assessing the disease severity in knee osteoarthritis patients? Int J Rheum Dis. 2017;20:1942-9.

12. Elbaz A, Magram-Flohr I, Segal G, Mor A, Debi R, Kalichman L. Association between knee osteoarthritis and functional changes in ankle joint and Achilles tendon. J Foot Ankle Surg. 2017;56(2):238-41.

13. Lee AC, Harvey WF, Price LL, Morgan LPK, Morgan NL, Wang C. Mindfulness is associated with psychological health and moderates pain in knee osteoarthritis. Osteoarthr Cartil. 2017:25(6):824-31.

14. Rundell SD, Goode AP, Suri P, Heagerty PJ, Comstock BA, Friedly JL, Gold LS, Bauer Z, Avins AL, Nedeljkovic SS, Nerenz DR, Kessler L, Jarvik JG. Effect of comorbid knee and hip osteoarthritis on longitudinal clinical and health care use outcomes in older adults with new visits for back pain. Arch Phys Med Rehabil. 2017;98(1):43-50.
15. Wright A, Benson HA, Will R, Moss P. Cold pain threshold identifies a subgroup of individuals with knee osteoarthritis that present with multimodality Hyperalgesia and elevated pain levels. Clin J Pain. 2017; 33(9):793.

16. Araujo IL, Castro MC, Daltro C, Matos MA. Quality of Life and Functional Independence in Patients with Osteoarthritis of the Knee. Knee Surg Relatated Res. 2016;28(3):219-24.

17. Bokaeian HR, Bakhtiary AH, Mirmohammadkhani M, Moghimi J. The effect of adding whole body vibration training to strengthening training in the treatment of knee osteoarthritis: a randomized clinical trial. J Bodyw Mov Ther. 2016:20(2):334-40.

18. Cho HJ, Gn KK, Kang JY, Suh KT, Kim TK. Epidemiological characteristics of patellofemoral osteoarthritis in elderly Koreans and its symptomatic contribution in knee osteoarthritis. Knee. 2016;23(1):29-34.

19. Kaban N, Tekeoğlu I, Harman H. Ultrasonographic characteristics of femoral trochlear cartilage in patients with knee osteoarthritis. Erciyes Tip Dergisi. 2016:38(4):127-60

20. Gomes-Neto M, Araujo AD, Junqueira ID, Oliveira D, Brasileiro A, Arcanjo FL. Comparative study of functional capacity and quality of life among obese and non-obese elderly people with knee osteoarthritis. Rev Bras Reumatol Engl Ed. 2016;56(2):126-30.

21. Khatib Y, Jenkin D, Naylor JM, Harris IA. Psychological traits in patients waiting for total knee arthroplasty. A cross-sectional study. J Arthroplast. 2016;31(8):1661-6.

22. Kiadaliri AA, Lamm CJ, de Verdier MG, Engstrom G, Turkiewicz A, Lohmander LS, Englund M. Association of knee pain and different definitions of knee osteoarthritis with health-related quality of life: a population-based cohort study in southern Sweden. Health Qual Life Outcomes. 2016;14(1):121.

23. Kiadaliri AA, Gerhardsson de Verdier M, Turkiewicz A, Lohmander LS, Englund M. Socioeconomic inequalities in knee pain, knee osteoarthritis, and health-related quality of life: a population-based cohort study in southern Sweden. Scand J Rheumatol. 2017;46(2):143-51.

24. Oishi K, Tsuda E, Yamamoto Y, Maeda S, Sasaki E, Chiba D, Ishibashi Y. The Knee injury and Osteoarthritis Outcome Score reflects the severity of knee osteoarthritis better than the revised Knee Society Score in a general Japanese population. Knee. 2016;23(1):35-42.

25. Sarumathy S, Balasudhakar C. Assessment of health-related quality of life and predictors of risk factors in patients with knee osteoarthritis. Int J PharmTech Res. 2016;9(5):461-5 ISSN: 0974-4304.

26. Cavalcante P, Doro M, Suzuki F, Rica R, Serra A, Pontes Junior F, Evangelista AL, Figueira Junior AJ. Functional fitness and self-reported quality of life of older women diagnosed with knee osteoarthritis: a cross-sectional case control study. J Aging Res. 2015;501:1-7.

27. Fang WH, Huang GS, Chang HF, Chen CY, Kang CY, Wang CC, Su SL. Gender differences between WOMAC index scores, health-related quality of life and physical performance in an elderly Taiwanese population with knee osteoarthritis. BMJ Open. 2015;5(9):e008542.

28. Ferreira AH, Godoy PBG, de Oliveira NRC, Diniz RAS, Diniz REAS, da Costa PR, Silva RC. Investigation of depression, anxiety and quality of life in patients with knee osteoarthritis: a comparative study. Revista Brasileira de Reumatologia (English Ed). 2015;55(5):434-8.

29. Kawano MM, Araújo ILA, Castro MC, Matos MA. Assessment of quality of life in patients with knee osteoarthritis. Acta Ortop Bras. 2015;23:307-10.

30. Kim HJ, Lee JY, Kim TJ, Lee JW. Association between serum vitamin D status and health-related quality of life (HRQoL) in an older Korean population with radiographic knee osteoarthritis: data from the Korean national health and nutrition examination survey (2010-2011). Health Qual Life Outcomes. 2015;13:48.

31. Lee S, Kim SJ. Prevalence of knee osteoarthritis, risk factors, and quality of life: the fifth Korean National Health and nutrition examination survey. Int J Rheum Dis. 2017;20(7):809-17. https://doi.org/10.1111/1756-185X.12795.

32. Pang J, Cao YL, Zheng YX, Gao NY, Wang XZ, Chen B, Gu XF, Yuan W. Influence of pain severity on health-related quality of life in Chinese knee osteoarthritis patients. Int J Clin Exp Med. 2015;8(3):4472-9.

33. Rakel B, Vance C, Zimmerman MB, Petsas-Blodgett N, Amendola A, Sluka KA Mechanical hyperalgesia and reduced quality of life occur in people with mild knee osteoarthritis pain. Clin J Pain. 2015:31:315-22.

34. Reid KF, Price LL, Harvey WF, Driban JB, Hau C, Fielding RA. Muscle power is an independent determinant of pain and quality of life in knee osteoarthritis. Arthritis Rheum. 2015;67:3166-73. 
35. Tsonga T, Michalopoulou M, Malliou P, Godolias G, Kapetanakis S, Gkasdaris $G$, Soucacos $P$. Analyzing the history of falls in patients with severe knee Osteorthritis. Clin Orthop Surg. 2015;7(4):449-56. https://doi.org/10.4055/ cios.2015.7.4.449.

36. Visser AW, de Mutsert R, Bloem JL, et al. Do knee osteoarthritis and fat-free mass interact in their impact on health-related quality of life in men? Results from a population-based cohort. Arthritis Care Res. 2015;67:981-8.

37. Alburquerque-García A, Rodrigues-de-Souza DP, Fernández-de-las-Peñas C, Alburquerque-Sendín F. Association between muscle trigger points, ongoing pain, function, and sleep quality in elderly women with bilateral painful knee osteoarthritis. J Manip Physiol Ther. 2015;38(4):262-8.

38. Alkan BM, Fidan F, Tosun A, Ardıçoğlu O. Quality of life and selfreported disability in patients with knee osteoarthritis. Mod Rheumatol. 2014;24:166-71.

39. Forkel P, Achtnich A, Metzlaff S, Zantop T, Petersen W. Midterm results following medial closed wedge distal femoral osteotomy stabilized with a locking internal fixation device. Knee Surg Sports Traumatol Arthrosc. 2015 23(7):2061-7.

40. Jahnke A, Mende JK, Maier GS, Ahmed GA, Ishaque BA, Schmitt H, Rickert M, Clarius M, Seeger JB. Sports activities before and after medial unicompartmental knee arthroplasty using the new Heidelberg sports activity score. Int Orthop. 2015;39(3):449-54.

41. Marks R. Perceived health status of women with knee osteoarthritis: a crosssectional study of the relationships of age, body mass, pain and walking limitations. Open Orthop J. 2014;8:255-63.

42. Perez-Prieto D, Gil-Gonzalez S, Pelfort X, Leal-Blanquet J, Puig-Verdie L, Hinarejos P. Influence of depression on total knee arthroplasty outcomes. J Arthroplast. 2014:29:44-7.

43. Reis JG, Gomes MM, Neves TM, Petrella M, de Oliveira RD, de Abreu DC. Evaluation of postural control and quality of life in elderly women with knee osteoarthritis. Rev Bras Rheumatol. 2014;54(3):208-12.

44. Alentorn-Geli E, Leal-Blanquet J, Guirro P, Hinarejos P, Pelfort X, Puig-Verdie L. Comparison of quality of life between elderly patients undergoing TKA. Orthopedics. 2013;356:e415-9.

45. Clement ND, Jenkins PJ, MacDonald D, Nie YX, Patton JT, Breusch SJ, Howie CR, Biant LC. Socioeconomic status affects the Oxford knee score and short-form 12 score following total knee replacement. Bone Joint J. 2013:95-B:52-8.

46. Vulcano E, Lee YY, Yamany T, Lyman S, Valle AG. Obese patients undergoing total knee arthroplasty have distinct preoperative characteristics: an institutional study of 4718 patients. J Arthroplast. 2013;28:1125-9.

47. Williams DP, Price AJ, Beard DJ, Hadfield SG, Arden NK, Murray DW, Field RE. The effects of age on patient-reported outcome measures in total knee replacements. Bone Joint J. 2013;95-B(1):38-44.

48. Coleman S, Briffa NK, Carroll G, Inderjeeth C, Cook N, McQuade J. A randomised controlled trial of a self-management education program for osteoarthritis of the knee delivered by health care professionals. Arthritis Res Ther. 2012;14(1):R21.

49. Goncalves RS, Pinheiro JP, Cabri J. Evaluation of potentially modifiable physical factors as predictors of health status in knee osteoarthritis patients referred for physical therapy. Knee. 2012;19:373-9. https://doi.org/10.1016/j. knee.2011.05.010.

50. Lim AT, Manching J, Penserga EG. Correlation between family APGAR scores and health-related quality of life of Filipino elderly patients with knee osteoarthritis. Int J Rheum Dis. 2012;15(4):407-13.

51. Elbaz A, Debbi EM, Segal G, Haim A, Halperin N, Agar G, Mor A, Debi R. Sex and body mass index correlate with Western Ontario and McMaster universities osteoarthritis index and quality of life scores in knee osteoarthritis. Arch Phys Med Rehabil. 2011;92:1618-23. https://doi.org/10. 1016/j.apmr.2011.05.009.

52. Gonçalves RS, Cabri J, Pinheiro JP. Evaluation of patient characteristics as predictors of health status in knee osteoarthritis patients referred for physical therapy. Acta Reumatol Port. 2011;36(2):137-44.

53. Norimatsu T, Osaki M, Tomita M, Ye Z, Abe $Y$, Honda S, Kanagae M, Mizukami S, Takamura N, Kusano Y, Shindo H, Aoyagi K. Factors predicting health-related quality of life in knee osteoarthritis among community-dwelling women in Japan: the Hizen-Oshima study. Orthopedics. 2011:34:e535-40.

54. Ozcakir S, Raif SL, Sivrioglu K, Kucukcakir N. Relationship between radiological severity and clinical and psychological factors in knee osteoarthritis. Clin Rheumatol. 2011;30(12):1521-6.
55. Paker N, Bugdayci D, Dere D. Determinants of quality of life in women with symptomatic knee osteoarthritis: the role of functional and emotional status. Turkish J Geriatr. Jan 2011;14(1):14-8.

56. Foroughi N, Smith RM, Lange AK, Baker MK, Singh MAF, Vanwanseele B. Dynamic alignment and its association with knee adduction moment in medial knee osteoarthritis. Knee. 2010;17:210-6.

57. Jenkins JB. Factors Influencing Quality of Life in Older Adults with Osteoarthritis Prior to Total Knee Replacement. Greensboro: Doctoral dissertation, University of North Carolina; 2010.

58. Kim I, Kim HA, Seo Yl, Song YW, Hunter DJ, Jeong JY, Kim DH. Tibiofemoral osteoarthritis affects quality of life and function in elderly Koreans, with women more adversely affected than men. BMC Musculoskelet Disord. 2010;11:129. https://doi.org/10.1186/1471-2474-11-129.

59. Muraki S, Akune T, Oka H, En-yo Y, Yoshida M, Saika A, Suzuki T, Yoshida H, Ishibashi H, Tokimura F, Yamamoto S, Nakamura K, Kawaguchi H, Yoshimura N. Association of radiographic and symptomatic knee osteoarthritis with health-related quality of life in a population-based cohort study in Japan: the ROAD study. Osteoarthr Cartil. 2010;18(9):1227-39.

60. Watanabe H, Urabe K, Takahira N, Ikeda N, Fujita M, Obara S, Hendona T, Aikawa J, Itoman M. Quality of life, knee function, and physical activity in Japanese elderly women with early-stage knee osteoarthritis. J Orthop Surg (Hong Kong). 2010;18:31-4

61. Yildiz N, Topuz O, Gungen GO, Deniz Z, Alkan H, Ardic F. Health - Related quality of life (Nottingham Health Profile) in knee osteoarthritis: correlations with clinical variables and self - reported disability. Rev Rheumatol Int. 2010; 30:1595-600.

62. Debi R, Mor A, Segal O, Segal G, Debbi E, Agar G, Halperin N, Haim A, Elbaz A. Differences in gait patterns, function, and quality of life between males and females with knee osteoarthritis: a clinical trial. BMC Musculoskelet Disord. 2009;10:127-37.

63. Imamura M, Imamura ST, Kaziyama HH, Targino RA, Hsing WT, de Souza LP, Cutait MM, Fregni F, Camanho GL. Impact of nervous system hyperalgesia on pain, disability, and quality of life in patients with knee osteoarthritis: a controlled analysis. Arthritis Rheum. 2008:59:1424-31. https://doi.org/10. 1002/art.24120.

64. Liikavainio T, Lyytinen T, Tyrvainen E, Sipilä S, Arokoski JP. Physical function and properties of quadriceps femoris muscle in men with knee osteoarthritis. Arch Phys Med Rehabil. 2008;89:2185-94.

65. Wang HM, Beyer M, Gensichen J, Gerlach FM. Health-related quality of life among general practice patients with differing chronic diseases in Germany: cross sectional survey. BMC Public Health. 2008;8:246. https://doi.org/10. 1186/1471-2458-8-246.

66. Nunez M, Nunez E, Segur JM, Maculé F, Sanchez A, Hernández MV, Vilalta C. Health-related quality of life and costs in patients with osteoarthritis on waiting list for total knee replacement. Osteoarthr Cartil. 2007;15:258.

67. Salaffi F, Carotti M, Stancati A, Grassi W. Health-related quality of life in older adults with symptomatic hip and KOA: a comparison with matched healthy controls. Aging Clin Exp Res. 2005;17(4):255-63.

68. Chacon JG, Gonzalez NE, Véliz A, Losada BR, Paul H, Santiago LG, Antúnez A Effect of knee osteoarthritis on the perception of quality of life in Venezuelan patients. Arthritis Rheum. 2004;51(3):377-82.

69. Lam CL, Lauder IJ. The impact of chronic diseases on the health-related quality of life (HRQOL) of Chinese patients in primary care. Fam Pract. 2000; 17:159-66. https://doi.org/10.1093/fampra/17.2.159.

70. de Leeuw JM, Villar RN. Obesity and quality of life after primary total knee replacement. Knee. 1998:5:119-23.

71. Donnell ST, Neyret P, Dejour H, Adeleine P. The effect of age on the quality of life after knee replacement. Knee. 1998;5:125-12.

72. Ries MD, Philbin EF, Groff GD. Relationship between severity of gonarthrosis and cardiovascular fitness. Clin Orthop Relat Res. 1995;313:169-76.

73. Witjes S, van Geenen RC, Koenraadt KL, van der Hart CP, Blankevoort L, Kerkhoffs GM, Kuijer PP. Expectations of younger patients concerning activities after knee arthroplasty: are we asking the right questions? Qual Life Res. 2017;26(2):403-17.

74. Skou ST. Efficacy of multimodal, systematic non-surgical treatment of knee osteoarthritis for patients not eligible for a total knee replacement: a study protocol of a randomised controlled trial. BMJ Open. 2012;2(6):e002168. https://doi.org/10.1136/bmjopen-2012-002168.

75. Skou ST, Roos EM. Good life with osteoArthritis in Denmark (GLA: D ${ }^{\text {TM}}$ ): evidence-based education and supervised neuromuscular exercise delivered 
by certified physiotherapists nationwide. BMC Musculoskelet Disord. 2017; 18(1):72. https://doi.org/10.1186/s12891-017-1439-y.

76. Jhun HJ, Sung NJ, Kim SY. Knee pain and its severity in elderly Koreans: prevalence, risk factors and impact on quality of life. J Korean Med Sci. 2013; 28(12):1807-13.

77. Pietersma S, de Vries M, van den Akker-van Marle ME. Domains of quality of life: results of a three-stage Delphi consensus procedure among patients, family of patients, clinicians, scientists and the general public. Qual Life Res. 2014;23(5):1543-56. https://doi.org/10.1007/s11136-013-0578-3.

78. Verges J, Vitaloni M, Bibas M, Sciortino R, Quintero M, Monfort J, Carné X, de Abajo F, Oswald E, Matucci M, du Souich P. Global OA management begins with quality of life assessment in knee OA patients: a systematic review. Osteoarthr Cartil. 2019;27:S229-30.

\section{Publisher's Note}

Springer Nature remains neutral with regard to jurisdictional claims in published maps and institutional affiliations.

Ready to submit your research? Choose BMC and benefit from:

- fast, convenient online submission

- thorough peer review by experienced researchers in your field

- rapid publication on acceptance

- support for research data, including large and complex data types

- gold Open Access which fosters wider collaboration and increased citations

- maximum visibility for your research: over $100 \mathrm{M}$ website views per year

At BMC, research is always in progress.

Learn more biomedcentral.com/submissions 